\title{
Decision Aids and Shared Decision-Making in Neurocritical Care: An Unmet Need in Our NeuroICUs
}

\author{
Susanne Muehlschlegel • Lori Shutter • \\ Nananda Col $\cdot$ Robert Goldberg
}

Published online: 6 January 2015

(c) Springer Science+Business Media New York 2014

\begin{abstract}
Improved resuscitation methods and advances in critical care have significantly increased the survival of patients presenting with devastating brain injuries compared to prior decades. After the patient's stabilization phase, families and patients are faced with "goals-of-care" decisions about continuation of aggressive intensive care unit care or comfort care only (CMO). Highly varying rates of CMO between centers raise the question of "self-fulfilling prophecies." Disease severity, the physician's communication and the family's understanding of projected outcomes, their uncertainties, complication risks with continued care, physician bias, and the patient's and surrogate's wishes and values all influence a CMO decision. Disease-specific decision support interventions, decision aids (DAs), may remedy these issues in the neurocritical care unit, potentially leading to better-informed and less-biased goals-of-care decisions in neurocritically ill patients, while increasing decision knowledge, confidence, and realistic expectations and decreasing decisional conflict and regret. Shared decision-
\end{abstract}

S. Muehlschlegel ( $\square)$

Departments of Neurology (Neurocritical Care), Anesthesia/ Critical Care and Surgery, University of Massachusetts Medical School, 55 Lake Ave. North, S5, Worcester, MA 01655, USA e-mail: susanne.muehlschlegel@umassmed.edu

\section{Shutter}

Departments of Critical Care Medicine, Neurology and Neurosurgery, University of Pittsburgh Medical Center, Pittsburgh, PA, USA

N. Col

Department of Medicine and Center of Excellence in Neuroscience, University of New England, Biddeford, ME, USA

R. Goldberg

Department of Quantitative Health Sciences, University of Massachusetts Medical School, Worcester, MA, USA making (SDM) is a collaborative process that enhances patients' and proxies' understanding about prognosis, encourages them to actively weigh the risks and benefits of a treatment, and considers the patient's preferences and values to make better decisions. DAs are SDM tools, which have been successfully implemented for many other conditions to assist difficult decision-making. In this article, we summarize the purposes of SDM, the derivation of DAs, and their potential application in neurocritical care.

Keywords Neurocritical care - Acute brain injury . Shared decision-making · Decision aid

Neurocritically ill patients with devastating neurological injuries most commonly die after withholding aggressive ICU care and shifting toward comfort care only (CMO) [1-3]. This process can lead to self-fulfilling prophecies $[2,4]$ and sometimes incite "clinical nihilism," which, as was highlighted in a review article by Hemphill and White [5], results in withholding aggressive interventions by clinicians based on the assumption that the patient is going to die anyways. While disease severity and patient preferences and values should drive the decision to shift towards CMO for critically ill patients, families often base CMO decisions on physician prognostication [6], making them subject to potential bias. To derive an estimate of a patient's prognosis, physicians commonly rely on pointbased prediction rules, past experiences, and personal biases $[2,7,8]$.

While disease-specific outcome models exist for the commonly encountered diseases in the neuro-intensive care unit (neuroICU), they may not always describe patientcentered outcomes, such as functional neurological outcome 
after survival. In addition, the communication of prognosis is not routinely taught during medical education, resulting in poor or complete avoidance of communication about prognosis and its uncertainties, as well as the assessment of patient and surrogate values and preferences $[9,10]$.

Qualitative research studies in families of critically ill traumatic brain injury (TBI) and all neuroICU patients have revealed that families often feel "under-informed" by physicians and nursing staff, partly due to mixed or biased messages from different providers and medical services [10-12]. Based on these studies, a higher family satisfaction and involvement in critical decision-making in the neuroICU is desirable.

Especially in patients with catastrophic neurological injuries, prognosis can be inherently biased and skewed toward an overly pessimistic or optimistic outlook [5]. As an example, in patients hospitalized with severe traumatic brain injury, recent investigations by members of the Canadian Clinical Trials group have revealed a disconcertingly high variability of CMO rates, ranging between 45 and $89 \%$, at six Canadian level I trauma centers [3]; this considerable variability was not explained by disease severity, age, or previously diagnosed co-morbidities. In a different study of a single Level I Trauma Center in the U.S., high variability in CMO recommendations between subspecialties involved in the care of patients with TBI (Trauma, Neurosurgery, Neurocritical Care, Anesthesiology/Surgical Critical Care) was also observed [4]. Two independently conducted surveys of intensivists, neurosurgeons, and neurologists exploring determinants of prognosis and clinical decision-making in adult patients with severe TBI revealed a significant variation in perceptions of neurologic prognosis, clinical decision-making, and recommendations to patient's families [4, 13]. Survey participants were given case vignettes and asked about the patient's prognosis 1 year later. In the Canadian study, approximately one-third of respondents agreed, one-third was neutral, and one-third disagreed that the patient's prognosis would be unfavorable at 1 year [13]. In a survey conducted at the U.S. Trauma Center, especially in younger patients, some clinicians prognosticated overly pessimistically based upon data available at the time of hospital admission [4]. Similarly high inter-hospital variability in early DNR orders (leading to CMO status) was found in patients after cardiac arrest [14] and intracerebral hemorrhage [15].

For neurointensivists, outcome prognostication is part of our daily work because of the nature and severity of patients admitted to neuroICUs. Therefore, the neuroICU serves as an ideal place to pilot decision support interventions that could improve and standardize the way prognosis and treatment options are communicated. At the same time, because critically ill patients with brain injury may not be able to express their wishes, and because decisions about treatment depend as much on a patient's prognosis as on patient values and preferences, it is equally important to assess these concerns and related issues via the patients' proxies as part of the decision-making process $[16,17]$.

Such decision support interventions have been termed "decision aids" (DAs) and are considered to be "shared decision making" tools. Shared decision-making is a collaborative process that enhances patients' and their proxies' understanding about the disease and its prognosis, encourages them to actively weigh the risks and benefits of a treatment, and assesses and matches this information to patient preferences and values, thereby decreasing decisional conflict and potentially improving decision quality and health outcomes. As two separate reviews have shown, patients want to be informed about their health condition, and many patients would like to participate in management of their disease $[16,18]$. Shared decision-making is a priority of the Patient Protection and Affordable Care Act of 2010 and Healthy People 2020. DAs have been successfully developed for many other conditions and diseases to assist with making difficult decisions and improve informed medical decision-making [19-21]. Examples are the liberation from the ventilator in generally critically ill patients with prolonged mechanical ventilation [22], discharge planning for patients admitted to a general intensive care unit ("Planning Care for Critically Ill Patients" [23] ), diabetes ("Should I take insulin?" [24]), or menopausal women with osteoporosis ("Healthy Bones" [25]).

Findings from the Cochrane Collaborative review of 86 randomized trials of DAs [19] show that they increase knowledge of treatment options and outcome probabilities, decision processes and quality, decrease decisional conflict, improve patient-practitioner communication, and increase medication adherence in the setting of various chronic diseases. Furthermore, and of pertinence to acute illnesses, DAs have been shown to improve accuracy of risk perception, increase knowledge about possible decisions to be made, change decisions about undergoing invasive procedures and elective surgery, and lead to more realistic expectations of treatment effects on disease outcomes. This is due in part to patients and proxies having heightened awareness and better understanding of the risks and benefits involved in making decisions.

Given this background, we propose that the use of validated DAs in the neuroICU for outcome prognostication and goals-of-care decisions may offer a more streamlined and standardized way of providing prognostication, discussing treatment approaches and setting correct expectations, all while limiting physician bias. In the neuroICU, these benefits may be particularly relevant for critically ill patients with catastrophic neurological injuries. The patient's impaired mental status precludes independent decision-making, and 
the surrogate is asked to make decisions on the patient's behalf. This introduces additional decision-making challenges and provides further opportunities for decision aids to support patient's values and preferences.

Several difficult areas in which DAs might be useful in the neuroICU include making decisions about tracheostomy, feeding tube placement, and implementation of Donot-resuscitate/Do-not-intubate orders. The most crucial decision involves the one surrounding goals-of-care, during which the physician asks the family or surrogate, based on the patient's prognosis, to decide about CMO or continuation of aggressive ICU care focusing on maximal survival. The latter commonly includes a tracheostomy with gastric feeding tube placement to help liberate the patient from the ventilator, followed by rehabilitation or admission to a nursing home. A DA, which supplements rather than replaces counseling by physicians, could be used to enhance patients' and proxies' understanding about prognosis [17] derived from validated prognostication models by illustrating statistical probabilities and uncertainties of outcome, (as well as potentially required surgical procedures) in a graphical and practical way. Visual aids, including the use of cartoons, symbols, photos, or videos, may play a large role in ensuring proper understanding of medical terms. For example, video DAs have been shown to effectively help patients and proxies [26] in making more informed decisions about end-of-life care and cardiopulmonary resuscitation in patients with dementia [27], advanced heart failure [28], cancer [29, 30], and critically ill patients with pulmonary disease [31]. Difficult treatment decisions are derived in concert with patient's values and preferences, which include their religious, cultural and ethnic beliefs, and prior experiences.

A recent Cochrane review concluded that for a DA to be effective and integrated into routine clinical care, it must contain disease-specific data that are tailored to patients and their proxies and be simple and time efficient for physicians to use $[17,19]$. To our knowledge, no DAs have been specifically developed nor tested for use with any of the difficult decisions commonly made in the neuroICU.

The development and validation of DAs can be complex. An internationally approved set of criteria to determine the quality of patient DAs have been published by the International Patient Decision Aid Standards (IPDAS) Collaboration [32] and should be rigorously applied to any newly developed DA. A toolkit for DA development [33] as well as large registries of existing DAs can be viewed online at the Ottawa Hospital Research Institute [21] and the Dartmouth-Hitchcock Center for Shared Decision-making [34].

The use of DAs could provide great benefit to critically ill patients in neuroICUs. When we extrapolate objectives previously achieved by DAs involving other diseases, such as cancer [19], to potential future DAs in the neuroICU, we see several likely benefits. Improved patient outcomes can be expected through better understanding of projected outcomes and risks, reduced decisional conflict, better quality decision-making processes with potentially reduced lengths-of-stay and costs (via more rapid transitions to either rehabilitation facilities or hospice), overall improved quality of care, and possibly limited physician bias. The development and implementation of disease-specific DAs in the neuroICU will enhance the ability of physicians with varying experience to more neutrally and objectively prognosticate and discuss treatment options while also eliciting patient's values and preferences. A neuroICU DA would be a standardized prognostication tool that is publicly available for all, simple to use, easy to understand, timesaving for the physician, and applicable during rounds or family meetings.

Conflict of interest Dr. Muehlschlegel and Dr. Col have nothing to disclose.

Funding Dr. Goldberg was funded by NIH/NHLBI for the Worcester Heart Attack Study (5R01HL035434-30). Dr. Shutter was funded by NIH/NINDS as a Site-PI for SHINE (1U10NS080371; Sub-Award \# GC12107-138778), co-investigator for NETT Pittsburgh Clinical Hub (5U10 NS080371-03), co-investigator for "Developing Goal-Directed Perfusion Therapy for Neurocardiac Injury in Subarachnoid Hemorrhage" (1R01NR014221-01A1), and Department of Defense as Co-investigator for "In Vivo Neuroimaging Biomarker Panel for Chronic Traumatic Encephalopathy" (W81XWH-13-2-0079).

\section{References}

1. Mayer SA, Kossoff SB. Withdrawal of life support in the neurological intensive care unit. Neurology. 1999;52:1602-9.

2. Becker KJ, Baxter AB, Cohen WA, et al. Withdrawal of support in intracerebral hemorrhage may lead to self-fulfilling prophecies. Neurology. 2001;56:766-72.

3. Turgeon AF, Lauzier F, Simard JF, et al. Mortality associated with withdrawal of life-sustaining therapy for patients with severe traumatic brain injury: a Canadian multicentre cohort study. CMAJ. 2011;183:1581-8.

4. Izzy S, Compton R, Carandang R, Hall W, Muehlschlegel S. Selffulfilling prophecies through withdrawal of care: do they exist in traumatic brain injury, too? Neurocrit Care. 2013;19:347-63.

5. Hemphill JC 3rd, White DB. Clinical nihilism in neuroemergencies. Emerg Med Clin North Am. 2009;27(27-37):7-8.

6. Johnston SC. Prognostication matters. Muscle Nerve. 2000;23:839-42.

7. Wilson ME, Rhudy LM, Ballinger BA, Tescher AN, Pickering BW, Gajic O. Factors that contribute to physician variability in decisions to limit life support in the ICU: a qualitative study. Intensive Care Med. 2013;39:1009-18.

8. Rabinstein AA, Hemphill JC 3rd. Prognosticating after severe acute brain disease: science, art, and biases. Neurology. 2010;74:1086-7.

9. Evans LR, Boyd EA, Malvar G, et al. Surrogate decision-makers' perspectives on discussing prognosis in the face of uncertainty. Am J Respir Crit Care Med. 2009;179:48-53. 
10. Bond AE, Draeger CR, Mandleco B, Donnelly M. Needs of family members of patients with severe traumatic brain injury. Implications for evidence-based practice. Critical Care Nurse. 2003;23:63-72.

11. Keenan A, Joseph L. The needs of family members of severe traumatic brain injured patients during critical and acute care: a qualitative study. Can J Neurosci Nurs. 2010;32:25-35.

12. Hwang DY, Yagoda D, Perrey HM, et al. Assessment of satisfaction with care among family members of survivors in a neuroscience intensive care unit. J Neurosci Nurs: J Am Assoc Neurosci Nurses. 2014;46:106-16.

13. Turgeon AF, Lauzier F, Burns KE, et al. Determination of neurologic prognosis and clinical decision making in adult patients with severe traumatic brain injury: a survey of Canadian intensivists, neurosurgeons, and neurologists. Crit Care Med. 2013;41:1086-93.

14. Richardson DK, Zive D, Daya M, Newgard CD. The impact of early do not resuscitate (DNR) orders on patient care and outcomes following resuscitation from out of hospital cardiac arrest. Resuscitation. 2013;84:483-7.

15. Hemphill JC 3rd, Newman J, Zhao S, Johnston SC. Hospital usage of early do-not-resuscitate orders and outcome after intracerebral hemorrhage. Stroke. 2004;35:1130-4.

16. Frosch DL, Kaplan RM. Shared decision making in clinical medicine: past research and future directions. Am J Prev Med. 1999;17:285-94.

17. Col NF. Chapter 17: Shared decision making. In: Fischoff B, Brewer NT, Downs JS, editors. Communicating risks and benefits: an evidence-based user's guide: US Department of Health and Human Services, Food and Drug Administration: Risk Communication Advisory Committee and consultants; 2011.

18. Benbassat J, Pilpel D, Tidhar M. Patients' preferences for participation in clinical decision making: a review of published surveys. Behav Med. 1998;24:81-8.

19. Stacey D, Legare F, Col NF, et al. Decision aids for people facing health treatment or screening decisions. Cochrane Database Syst Rev. 2014;1:CD001431.

20. Institute OHR. Ottawa Hospital Research Institute: cochrane decision aid registry. 2011. http://decisionaid.ohri.ca/cochinvent.php. Accessed 23 Aug 2014.

21. Ottawa Hospital Research Institute. Patient decision aids. http:// decisionaid.ohri.ca/index.html. Accessed 23 Aug 2014.

22. Cox CE, Lewis CL, Hanson LC, et al. Development and pilot testing of a decision aid for surrogates of patients with prolonged mechanical ventilation. Crit Care Med. 2012;40:2327-34.
23. Kryworuchko J. Understanding the options: planning care for critically ill patients in the intensive care unit. The Foundation for Informed Medical Decision Making. 2009. http://decisionaid. ohri.ca/docs/das/Critically_Ill_Decision_Support.pdf. Accessed 23 Aug 2014.

24. Staff H. Decision aid: should I take insulin? Healthwise. 2014. http://www.healthwise.net/cochranedecisionaid/Content/StdDocu ment.aspx?DOCHWID=abo2664. Accessed 23 Aug 2014.

25. White E, Volk R, Nelson B, et al. Healthy bones. 2011. http://www. effectivehealthcare.ahrq.gov/ehc/decisionaids/osteoporosis/. Accessed 23 Aug 2014.

26. Volandes AE, Mitchell SL, Gillick MR, Chang Y, Paasche-Orlow MK. Using video images to improve the accuracy of surrogate decision-making: a randomized controlled trial. J Am Med Dir Assoc. 2009;10:575-80.

27. Volandes AE, Paasche-Orlow MK, Barry MJ, et al. Video decision support tool for advance care planning in dementia: randomised controlled trial. BMJ. 2009;338:b2159.

28. Veroff DR, Sullivan LA, Shoptaw EJ, et al. Improving self-care for heart failure for seniors: the impact of video and written education and decision aids. Popul Health manag. 2012;15:37-45.

29. Epstein AS, Volandes AE, Chen LY, et al. A randomized controlled trial of a cardiopulmonary resuscitation video in advance care planning for progressive pancreas and hepatobiliary cancer patients. J Palliat Med. 2013;16:623-31.

30. El-Jawahri A, Podgurski LM, Eichler AF, et al. Use of video to facilitate end-of-life discussions with patients with cancer: a randomized controlled trial. J Clin Oncol. 2010;28:305-10.

31. McCannon JB, O'Donnell WJ, Thompson BT, et al. Augmenting communication and decision making in the intensive care unit with a cardiopulmonary resuscitation video decision support tool: a temporal intervention study. J Palliat Med. 2012;15:1382-7.

32. International Patient Decision Aids Standards (IPDAS) Collaboration. Update of the IPDAS collaboration background document. 2012. http://ipdas.ohri.ca/resources.html. Accessed 23 Aug 2014.

33. Ottawa Hospital Research Institute. Decision aid development tool kit. https://decisionaid.ohri.ca/resources.html. Accessed 18 June 2014.

34. Dartmouth-Hitchcock Center for Shared Decision Making Decision Aid Library. http://patients.dartmouth-hitchcock.org/shared_ decision_making/decision_aid_library.html. Accessed 18 June 2014. 